\title{
Treatment-free remission in patients with chronic myeloid leukemia
}

\author{
Delphine $\operatorname{Rea}^{1} \cdot$ Jean-Michel Cayuela $^{2}$
}

Received: 27 April 2017 / Accepted: 1 May 2017 / Published online: 8 July 2017

(C) The Japanese Society of Hematology 2017

\begin{abstract}
Clinical trials have formally demonstrated that in chronic myeloid leukemia (CML), patients treated with tyrosine kinase inhibitors (TKI) who achieved and maintained deep molecular responses could discontinue their treatment after several years without facing overt signs of disease relapse in approximately $50 \%$ of the cases. In patients with a molecular relapse, prompt re-introduction of TKI therapy was able to rapidly restore deep molecular responses. The concept of a lifelong therapy with TKI has thus been challenged and treatment-free remission (TFR) strategies will soon integrate clinical practice, providing that safe recommendations will be established. In this article, we give an update on TKI discontinuation studies in CML and we also provide an overview of upcoming TFR clinical and biological challenges.
\end{abstract}

Keywords Tyrosine kinase inhibitors · Treatment discontinuation $\cdot$ Chronic myeloid leukemia

\section{Introduction}

Imatinib mesylate, the first ATP-competitive tyrosine kinase inhibitor (TKI) of BCR-ABL1, the driving oncoprotein of chronic myeloid leukemia (CML), received

Delphine Rea

delphine.rea@aphp.fr

1 Service d'hématologie Adulte, Unité INSERM, UMR-1160 and France Intergroupe des Leucémies Myéloïdes Chroniques (Fi-LMC), Hôpital Saint-Louis, 1 Avenue Claude Vellefaux, 75010 Paris, France

2 Laboratoire Central d'Hématologie, EA3518 Université Paris 7 and France Intergroupe des Leucémies Myéloïdes Chroniques (Fi-LMC), Hôpital Saint-Louis, Paris, France approval for use in adult patients in the early 2000s based on a dramatic and durable overall and progression-free survival benefit $[1,2]$. Subsequently, other TKI with a greater target-specific potency and varying off-target inhibition profiles were designed and tested with the goal to fight mutation-driven resistance and drug-related intolerance affecting about one-third of imatinib-treated patients [3]. The second generation compounds dasatinib, nilotinib and bosutinib and the third generation drug ponatinib became part of the therapeutic arsenal against the disease and allogeneic hematopoietic stem cell transplantation was relegated to a position of last resort option despite its curative potential $[4,5]$.

Life expectancy of adult patients with CML who optimally respond to TKI therapy is approaching that of the general population and the prevalence of the disease increases overtime [6,7]. Despite these major advances, ATP-competitive TKIs are considered as non-definitively curative, due to their inability to eradicate quiescent $B C R$ $\mathrm{ABLI}^{+}$leukemia stem cells despite inhibition of BCRABL1 activity. Indeed, these cells do not depend on BCRABL1 for their survival and they represent a reservoir fully capable of restoring CML as attested by leukemic cell expansion in murine models and upon TKI withdrawal in most patients, justifying the recommendation to pursue treatment lifelong [8-12]. However, a pilot study performed more than 10 years ago in patients with so-called "molecularly undetectable disease" for prolonged period of times challenged the statement that TKI may never be stopped [13]. In the subsequent prospective STIM and TWISTER trials, imatinib discontinuation was proposed after a minimum of 3 years of treatment on condition that patients had achieved an at least $4.5 \log$ reduction in $B C R-A B L 1^{+}$residual disease and that $B C R-A B L 1$ transcripts were undetectable for at least 2 years $[14,15]$. In these highly selected 


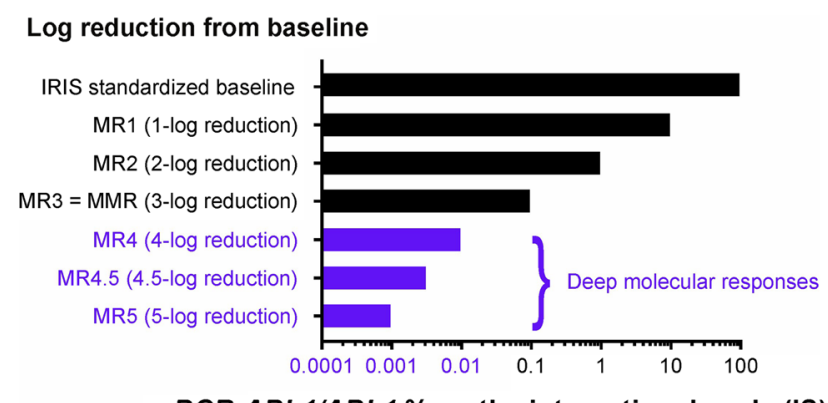

$B C R-A B L 1 / A B L 1 \%$ on the international scale (IS)

Fig. 1 Definition of deep molecular responses. Deep molecular responses (shown in blue) are defined as follows: MR4 ( $\geq 4-\log$ reduction from IRIS baseline) $=$ either detectable disease with $B C R$ $A B L 1 \leq 0.01 \%$ IS or undetectable disease with $10000-<32000 A B L 1$ transcripts. MR4.5 ( $\geq 4.5-\log$ reduction from IRIS baseline) $=$ either detectable disease with $B C R-A B L 1 \leq 0.0032 \%$ IS or undetectable disease with $32000-<100000 A B L 1$ transcripts. MR5 ( $\geq 5$ - $\log$ reduction from IRIS baseline) $=$ either detectable disease with $B C R-A B L 1$ $\leq 0.001 \%$ IS or undetectable disease with $\geq 100000 A B L 1$ transcripts. When using other control genes than $A B L 1$ and in case of undetectable disease, copy numbers of control gene transcripts used to define deep molecular responses must be adapted [17] patient populations, the 2-year probability to maintain such deep molecular response levels without any treatment was $38 \%$ in STIM and $47 \%$ in TWISTER [14, 15]. Although several independent studies provided evidence that leukemic stem cells persisted in most, if not all, imatinib-treated patients regardless of outcome after treatment discontinuation, follow-up analyses of the 100 patients enrolled in STIM proved long-term stability of TFR [16]. In the meantime, important efforts were made in order to better standardize and score deep molecular responses at different levels of RT-qPCR sensitivity (Fig. 1) [17]. Discontinuation of new generation TKI was also investigated.

TFR is on the way to become an important goal in clinical practice, implicating a change in CML management principles in the near future (Fig. 2). The aim of this article is to provide update on TKI discontinuation studies and an overview of upcoming clinical and biological challenges.

\section{Treatment-free remission studies: imatinib}

Following STIM and TWISTER, several independent studies confirmed that imatinib-free remission was possible

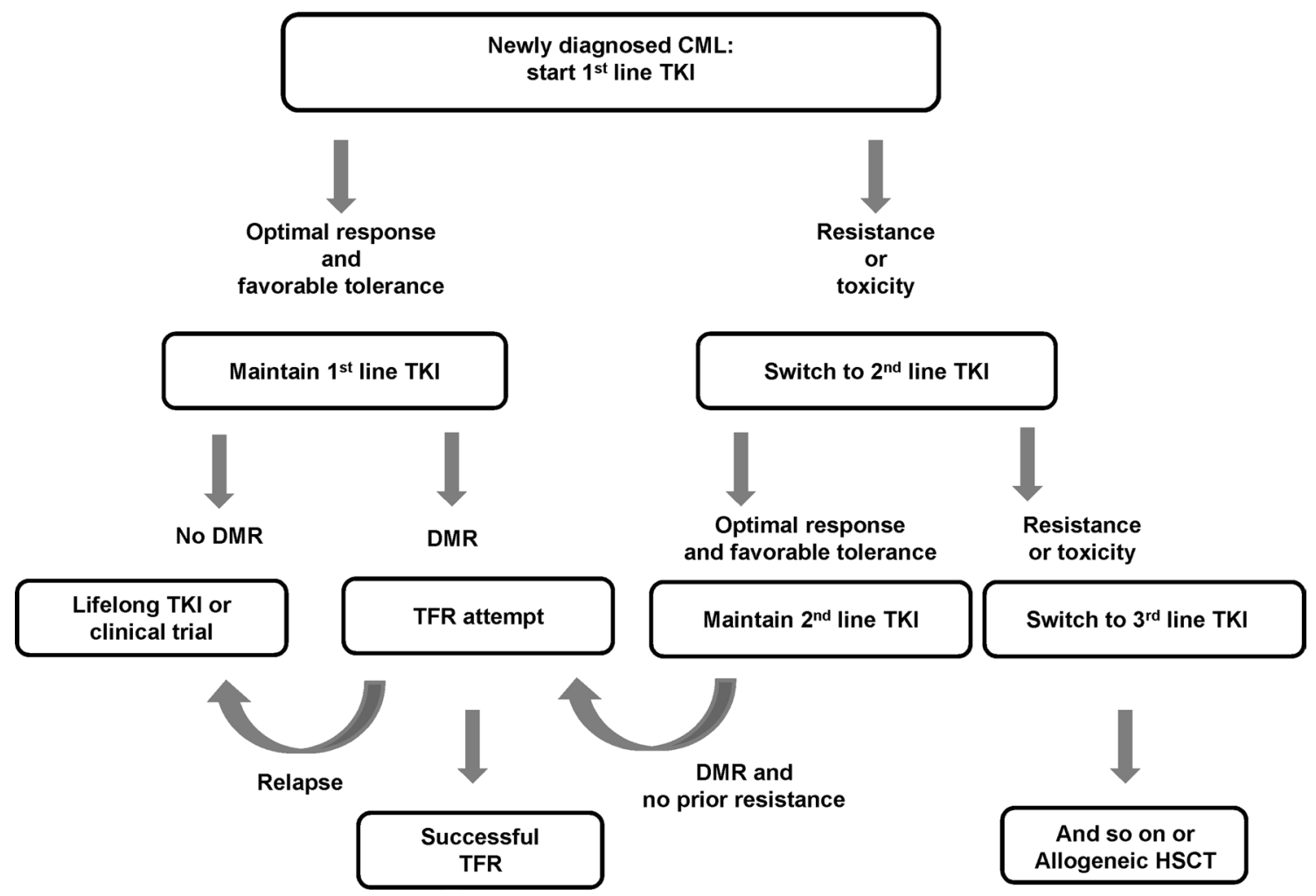

Fig. 2 Principle of CML management integrating TFR in clinical practice. Patients achieving and maintaining deep molecular responses (DMR) during first or subsequent line TKI treatment in the absence of prior TKI resistance may be offered a treatmentfree remission attempt in clinical practice. In case of a molecular relapse, TKI should be reintroduced and a second TKI discontinua- tion attempt should only be envisaged in the context of clinical trials. Patients who do not obtain deep molecular responses may also be candidates for clinical trials. Safe and appropriate criteria for TKI discontinuation in clinical practice should follow evidence-based recommendations 
in patients with deep and durable molecular responses (Table 1). The observational according to STIM (A-STIM) study was the first to show that the loss of a major molecular response (MMR), a less stringent definition of molecular relapse than in STIM and TWISTER, represented a safe trigger for TKI resumption [18]. In this study, $80 \mathrm{CML}$ patients stopped imatinib after a median time on therapy of 79 months (range 30-145) and a median duration of MR4.5 of 41 months (range 24-96). TFR estimate was $64 \%$ (95\% CI 54-75) at 12 months and $61 \%$ at 24 months (95\% CI 51-73). The majority of MMR loss occurred soon after imatinib discontinuation and among the 29 relapsing patients, only one lost MMR after 12 months. Importantly, peripheral blood $B C R-A B L 1$ transcripts could be detected in 55\% of non-relapsing patients either in an intermittent fashion or in a more continuous way, thus demonstrating that low levels of $B C R-A B L 1$ transcripts after TKI discontinuation was not automatically associated with progressive CML.

The multicenter prospective Korean Imatinib Discontinuation Study (KIDS) primarily aimed at evaluating the probability of sustained MMR after imatinib discontinuation and thus used MMR loss as molecular relapse criterion as in A-STIM [19, 20]. Ninety patients stopped imatinib after a median duration of treatment of 80.8 months (range 38.2-141.3) and a median duration of MR4.5 with undetectable $B C R-A B L 1$ transcripts of 39.9 months (range 22.1-130.7). As in other imatinib discontinuation studies, the median time to MMR loss in the 37 relapsing patients was short (median 3.3 months, range 0.9-20.8). The rate of sustained MMR without any treatment was close to that obtained in A-STIM, with 12- and 24-month probabilities of 62.2 and $58.5 \%$, respectively.

The main goal of the European multicenter study EURO-SKI performed under the sponsorship of the European Leukemia Net (ELN) was to assess of the duration of MMR or better after stopping TKI therapy in a very large population of CML patients (ClinicalTrials.gov Identifier: NCT01596114). Key eligibility criteria were less stringent than in prior imatinib discontinuation studies and included a total duration of TKI therapy of at least 3 years and MR4 duration of at least 1 year. EURO-SKI excluded patients with a history of resistance to TKI. As in A-STIM, loss of MMR defined molecular relapse. The final analysis of this study in which 821 patients were included has not been performed yet, but preliminary results were presented on several occasions and most patients stopped first line imatinib. Survival without molecular relapse (MRFS) was assessed in 755 patients with a median duration of TKI therapy of 7.5 years (range 3-14.2) and a median duration of MR4 of 4.7 years (range 1-13.3). Unlike in other TKI discontinuation studies, MRFS probabilities were continuously decreasing, being at $61 \%$ (95\% CI 58-75), 55\% (95\% CI 
51-58), 50\% (95\% CI 47-54) and 47\% (95\% CI 43-51) at $6,12,24$ and 36 months, respectively [21]. The reason for this steady decrease in TFR probabilities overtime is currently unknown and may relate to a between-patients variability of deep molecular response levels prior to TKI discontinuation and to the choice of MR4 and not MR4.5 as a threshold to discontinue therapy.

\section{Treatment-free remission studies: dasatinib and nilotinib}

Experience about second generation TKI discontinuation is quiet recent but results from several studies evaluating dasatinib or nilotinib-free molecular remission are already available (Table 2). These studies are of particular interest because these 2 TKI are not solely useful to rescue patients facing imatinib failure or intolerance [22, 23]. Indeed, a switch to second line nilotinib increases the probability to achieve deep molecular responses compared to imatinib maintenance in patients with a complete cytogenetic response (CCyR) on imatinib [24]. Moreover, first line dasatinib or nilotinib in newly diagnosed CP-CML induces a more rapid decline in $B C R-A B L 1$ transcripts and significantly higher rates of deep molecular responses compared with imatinib at $400 \mathrm{mg}$ daily [25, 26]. Altogether, these data suggest that dasatinib or nilotinib may increase access to TFR strategies as compared to standard dose imatinib. Nevertheless, there is currently no evidence of improved TFR rates after dasatinib or nilotinib discontinuation compared with what reported for imatinib, although it should be recognized that no randomized comparison exists.

The phase 2 Japanese multicenter Dasatinib Discontinuation trial (DADI) investigated TFR in patients treated with second or subsequent line dasatinib and in deep molecular response defined as a MR4 with detectable or undetectable $B C R-A B L 1$ transcripts [27]. One year of MR4 consolidation with dasatinib therapy was given prior to the TFR phase which included 63 patients. All molecular relapses defined as loss of MR4 on a single occasion occurred soon after dasatinib discontinuation. After a median follow-up of 20 months, the TFR probability was $48 \%$ (95\% CI 35-59) at 12 months and remained stable at later time points.

First or subsequent line dasatinib discontinuation was also evaluated in the context of the international multicenter DASFREE trial in 84 patients with at least 2 years of dasatinib treatment and in MR4.5 for at least 1 year (ClinicalTrials.gov Identifier: NCT01850004). A first interim analysis performed on 30 patients revealed a molecular relapse free survival probability of $63 \%$ at 12 months, molecular relapse being defined as the loss of MMR [28]. Updated results and a total follow-up of 5 years are planned. The French multicenter STOP 2G-TKI observational study evaluated first or subsequent line dasatinib or nilotinib discontinuation in patients with at least 3 years of TKI treatment and a deep molecular response duration of at least 2 years. Deep molecular response was defined as a MR4.5 with undetectable $B C R-A B L 1$ transcripts and molecular relapse was defined as the loss of MMR on a single occasion. A first interim analysis reported outcomes of 60 patients with a minimum follow-up of 12 months and a median follow-up of 47 months (range 12-65). TFR rates at 12 and 48 months were $63.33 \%$ (95\% CI 51.14-75.53) and $53.57 \%$ (95\% CI 40.49-66.65), respectively [29]. As observed in DADI, the majority of molecular relapses $(81 \%)$ occurred within 12 months after treatment cessation.

The phase 2 international multicenter trial ENESTop investigated second line nilotinib discontinuation in patients treated with TKI for at least 3 years including 2 years at least of nilotinib and in whom a deep molecular response defined as a MR4.5 was achieved during nilotinib treatment (ClinicalTrials.gov Identifier: NCT01698905). One year of MR4.5 consolidation with nilotinib was given prior to treatment discontinuation and 126 patients in stable MR4.5 entered the TFR phase. Molecular relapse was defined as the loss of MMR on a single occasion or a confirmed loss of MR4. The follow-up is still short and TFR rate at 48 weeks was $58.7 \%$ (95\% CI 49.6-66.7) [30]. Follow-up of patients is planned for up to 4 years.

The phase 2 international multicenter trial ENESTfreedom studied upfront nilotinib discontinuation in patients treated for at least 2 years and in deep molecular response defined as a MR4.5 (ClinicalTrials.gov Identifier: NCT01784068). After 1 year of consolidation with nilotinib and a median duration of nilotinib treatment of 43.5 months, 190 patients in stable MR4.5 entered the TFR phase. Molecular relapse was defined as the loss of MMR on a single occasion. The follow-up is still short and the probability to remain in TFR at 48 weeks was $51.6 \%$ (95\% CI 44.2-58.9) [31]. Patient follow-up is planned for up to 4 years. Other multicenter nilotinib or dasatinib discontinuation studies are currently ongoing including D-STOP (ClinicalTrials.gov Identifier: NCT01627132), NILSt, STAT2 or ENESTpath (ClinicalTrials.gov Identifier: NCT01743989) [32-35].

\section{Patient safety in TFR studies}

Overall, death due to CML was not reported in TFR studies and major safety issue was extremely rare. A highfrequency molecular monitoring was performed for early detection of molecular relapses, usually on a monthly basis during the first year and every 2-3 months thereafter. Patients who experienced a molecular relapse were instructed to rapidly restart the same TKI as prior to discontinuation unless otherwise medically indicated. Deep molecular responses were rapidly regained, indicating that 


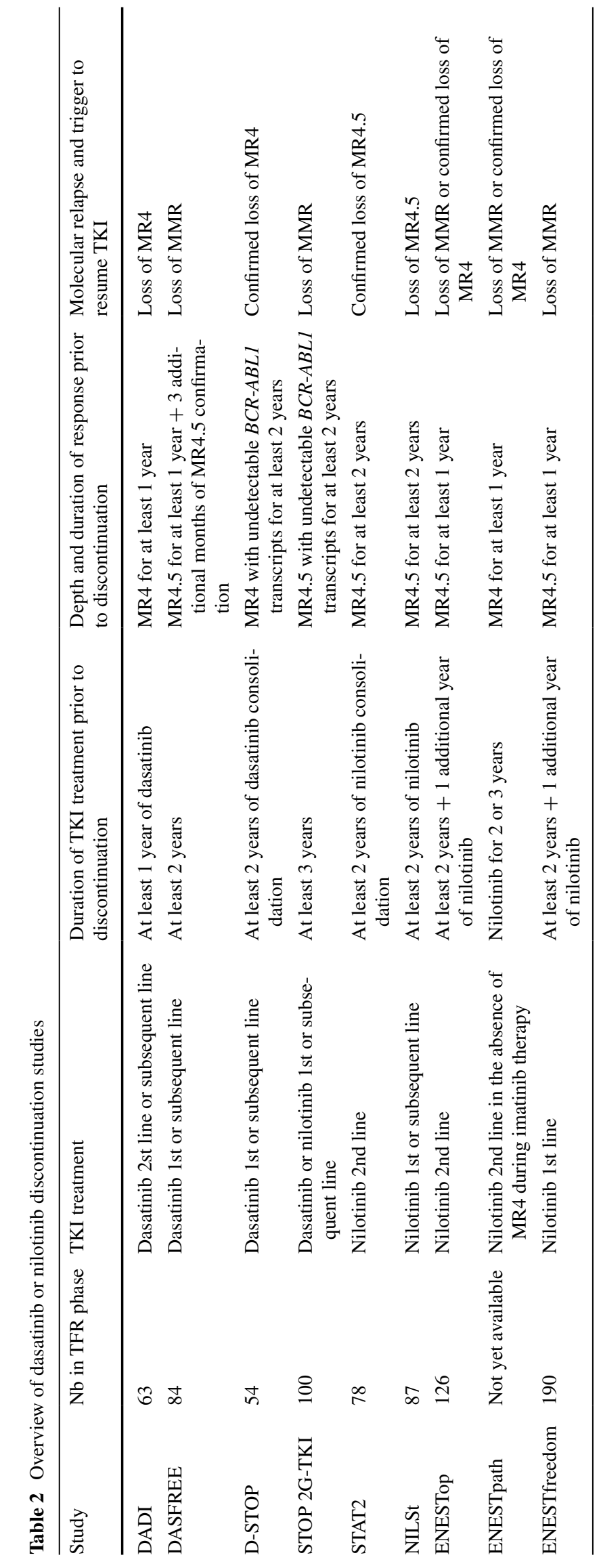


TKI discontinuation trials performed under stringent monitoring conditions and treatment resumption policies did not favor the emergence of secondary resistance (Fig. 3). However, there were two exceptions to these reassuring data. First, MMR loss was accompanied by the presence of a nilotinib-resistant F359V BCR-ABL1 mutation in a patient enrolled in ENESTfreedom [31]. Whether this mutant clone preexisted at a very low level prior to the TFR phase or emerged after nilotinib discontinuation could not be explored [36]. Second, CML transformation into lymphoid blast phase was described in a patient who lost MMR after imatinib discontinuation in the A-STIM study [18]. Intriguingly, transformation did not occur during the TFR phase, but 8.5 months after imatinib was restarted and after a MMR was regained. No imatinib-resistant mutation was identified, but cytogenetic analyses revealed chromosomal abnormalities in addition to the Philadelphia chromosome. Whether the $B C R-A B L 1^{+}$lymphoid leukemic clone was selected during imatinib treatment, during the TFR phase or after imatinib resumption remains unknown. In any case, this situation resembles sudden blast crisis that may exceptionally occur either in optimal responders to IFN- $\alpha$ or TKI or after IFN- $\alpha$ cessation or allogeneic stem cell transplantation [37-39]. Although a formal link with TKI discontinuation cannot be established, caution is needed in ongoing and future studies. Determining the exact frequency of such rare disease-related life-threatening event is a key issue from both patients and physicians perspective.

A logical expectation in TFR studies consists in the disappearance of TKI-related side effects during the treatment-free phase and indeed, most drug-related adverse events regress after imatinib discontinuation [40]. Unexpectedly, pruritus and newly occurring or worsening musculoskeletal pain within several weeks after TKI discontinuation was reported in about $30 \%$ of patients [20, 41]. This reversible phenomenon called the "TKI withdrawal syndrome" can last for up to several months. Musculoskeletal pain may necessitate prescription of painkillers such as paracetamol or nonsteroidal or anti-inflammatory drugs, less frequently a course of corticosteroids. Its pathophysiological mechanism is unknown. It rapidly disappears in patients who restart TKI therapy after a molecular relapse. Whether this "withdrawal syndrome" may be minimized by tapering TKI doses over several months before discontinuation is an open question. Other aspects of patient safety such as clinically relevant effects of the suppression of TKI-other drug interactions upon TKI discontinuation have not been looked at.

\section{Prognostic factors of TFR}

There is a high clinical need in trying to identify factors that significantly impact the likelihood of succeeding TKI discontinuation in order to minimize potential risks of a leukemic rebound, to avoid undesirable drug-withdrawal symptoms, not to disappoint patient expectations and to prevent unnecessary overuse of medical tests [41, 42]. So far, the search for clinical variables associated with outcome has been challenging. Keeping in mind the limitations due to varying trial design, factors such as the Sokal score, duration of therapy and duration of deep molecular response have provided some insights into the probability of successful imatinib discontinuation [14, 16, 20,43].

In STIM, prognostic variable analyses showed that a low or intermediate Sokal score and a long duration of imatinib therapy were associated with best TFR probabilities [16]. Using cut-off optimization methods, it was shown that patients who had been treated with imatinib for at least 54 months had a significantly lower probability of molecular relapse than patients with a shorter duration of
Fig. 3 Evolution of $B C R-A B L 1$ transcripts levels in a representative individual patient. $B C R-A B L 1$ transcripts levels as measured by RT-qPCR from TKI introduction after CML diagnosis until TKI re-introduction upon MMR loss after treatment discontinuation

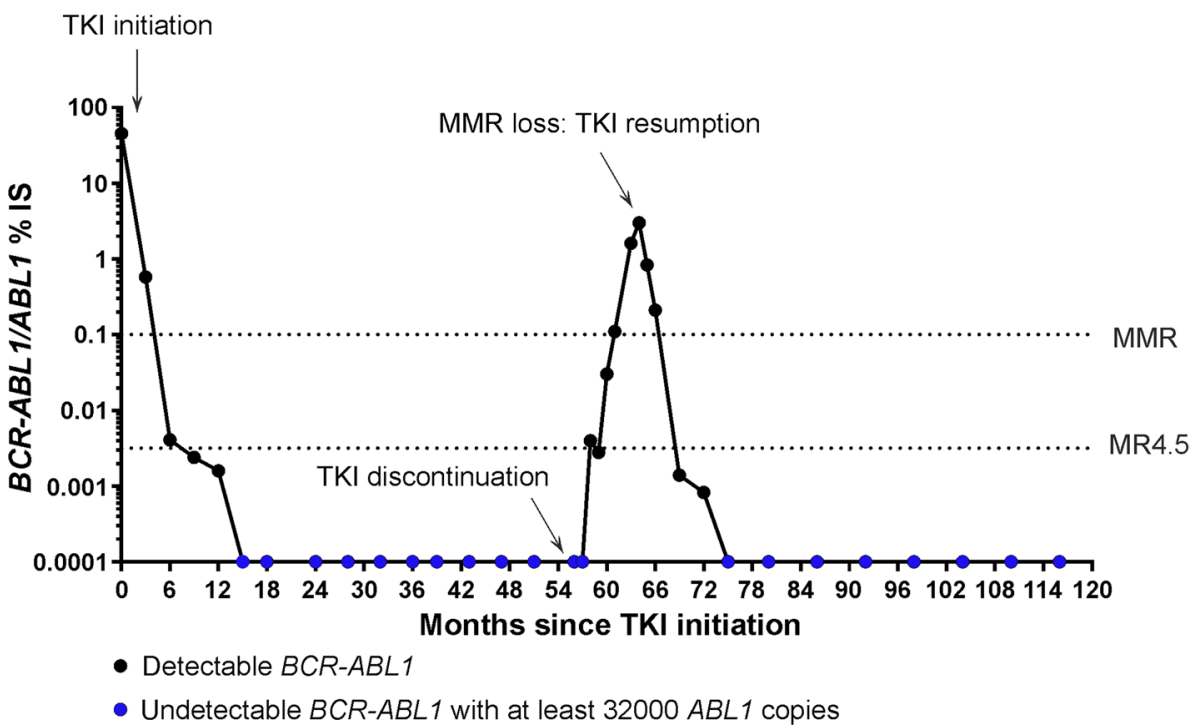


imatinib. Analyses performed in EURO-SKI also found a detrimental effect of a short duration of imatinib treatment and a short duration of a deep molecular response prior to treatment discontinuation on TFR [21]. EUROSKI also aimed at determining whether the varying levels of deep molecular responses may influence TFR probabilities but this question may benefit from the use of new technologies with a better quantitative accuracy than RT-qPCR such as digital PCR [21, 43].

It is too early yet to know whether the same factors influence TFR rates in new generation TKI discontinuation studies but both in DADI and in STOP 2G-TKI, a history of suboptimal response or resistance to imatinib was associated with a significantly reduced probability of achieving a TFR after dasatinib or nilotinib discontinuation [27, 29]. There is no obvious explanation for this observation since all patients were in deep and durable molecular response before therapy discontinuation, but it may be resolved as we gain more knowledge on the inter-individual functional heterogeneity of the leukemic stem cells reservoir [44].

\section{Real life experience with TKI discontinuation}

The fundamental treatment goal in CML treatment is to protect patients from progression to blast crisis, such an event being a challenging issue as long-term survival is rarely achieved even with TKI combined to chemotherapy followed by allogeneic stem cell transplantation. However, there are many reasons to also aim at TFR rather maintaining TKI therapy indefinitely [45]. TFR offers the obvious advantages of getting rid of chronic side effects of TKI, avoiding potentially severe toxicities especially during pregnancy and in elderly or comorbid patients, and reducing expenses borne by individuals or health care systems $[46,47]$. There have been few reports on TKI discontinuation in the real-life setting. Takahashi et al. conducted a nationwide survey in Japan and found that among 43 imatinib-treated patients with undetectable $B C R-A B L 1$ transcripts (RT-qPCR sensitivity of at least 4-log) for a median time of 27.4 months (range 0.9-79.6), the 5-year molecular recurrence-free survival rate after imatinib discontinuation was $47 \%$ [48]. Benjamini et al. analyzed outcome after patient-driven TKI discontinuation and found that among 27 patients with undetectable $B C R-A B L 1$ transcripts (RT-qPCR sensitivity of at least $5-\log$ ), the probability to maintain such deep molecular response levels at 12 months was $60.7 \%$ (95\% CI 42.3-7.1) [49]. RegeCambrin et al. studied outcome of 42 patients with undetectable $B C R-A B L 1$ transcripts (RT-qPCR sensitivity of at least 4-log) for a median time of 37 months (range 3-56) who discontinued imatinib and the MR4 relapse-free survival rate at 12 months was $57.7 \%$ (95\% CI 44.7-74.2) [50]. Kong et al. focused on patients who discontinued TKI therapy for any reason while in deep molecular response (defined as $B C R-A B L 1$ transcripts $<0.0063 \%$ IS) for at least 2 years and using MMR loss as a trigger for therapy resumption, found that TFR probabilities at 1 and 2 years were $65.7 \%$ (95\% CI 55.8-75.6) and 59.7\% (95\% CI 49.170.3), respectively [51]. No safety issues were reported in these studies and data suggest that patient outcome may not substantially differ from that of patients enrolled in clinical trials. However, these studies were retrospective in design and physician reluctance to include patients with serious safety issues after TKI discontinuation cannot be excluded. Clear and pragmatic recommendations on TKI discontinuation using rigorous evidence-based methodology will emerge in the near future with the goal to determine if, when and how patients may safely try to stop TKI in clinical practice. Patient concerns regarding potential disease recurrence as well as patient adherence to TFR strategies including monitoring and treatment resumption policies are two other key aspects to be taken into consideration for clinical decision making.

\section{Areas of research and future prospects}

So far, results from several clinical trials in CP-CML patients treated upfront with imatinib at $400 \mathrm{mg}$ QD indicate that the incidence of deep molecular responses, a prerequisite for TFR, is quiet low. In DASISION and ENESTnd, the cumulative incidence of MR4.5 in each trial imatinib arm was 33 and $31 \%$ by 5 years, respectively $[25,26]$. In the IRIS study, analysis of long term outcomes showed a MR4.5 rate at 5 and 10 years calculated on an intention to treat basis of $23.3 \%$ [2]. A study performed in 93 imatinib-treated patients in the real life setting found that the proportion of patients who obtained a deep molecular response such as a MR4 or a MR4.5 lasting for at least 2 years was 28\% [52]. Dasatinib or nilotinib given upfront or in patients lacking deep molecular responses on imatinib significantly increase the likelihood of deep molecular responses [24-26]. However, there is still a long road before all patients may be eligible for TFR and a large room for improvement in TFR success rates. Having in mind the potential effect of IFN- $\alpha$ on CML stem cells, at least in murine models, and the superiority of imatinib + IFN- $\alpha$ over imatinib alone in terms of deep molecular responses induction, randomized studies are underway in order to evaluate the deep molecular response induction potential of second generation TKI associated with pegylated IFN [5355]. However, TFR rates for patients stopping TKI+IFN- $\alpha$ combination therapies have not yet been investigated. Following a pilot study in patients who unsuccessfully stopped imatinib, successive TKI discontinuation attempts are also becoming an area of research [56]. In such patients, testing add-on treatments to standard TKI rationally chosen 
for their anti-leukemic stem cell effects may be of particular interest. While there is a broad consensus about leukemic stem cell persistence in all patients even those in deep molecular responses, the reasons underlying divergent outcome after TKI discontinuation is unknown [57]. The immune system may contribute to long-term TFR durability as several independent studies highlighted a relationship between TFR probabilities and immune effectors such as NK-cells, although a direct role of such cells has not been formally proven $[27,58,59]$. There may be substantial quantitative or qualitative heterogeneity in the leukemic stem cell reservoir and its niche between patients but such investigations are hampered by technical difficulties.

\section{Conclusion}

Demonstration from multiple studies that TKI discontinuation is feasible and safe in patients with deep and durable molecular responses on-therapy has challenged the concept of a lifelong treatment in CML. TFR is about to be integrated into clinical practice, although optimal selection criteria for such a strategy are still a matter of debate and there is a need for consensus recommendations to guide both physicians and patients. Patient awareness about what is certain or uncertain should be an important part of informed decision making. Clinical trials are still needed since many unanswered questions remain, as well as biological investigations aiming at developing approaches to eradicate LSC, in the idea of curing the disease.

\section{Compliance with ethical standards}

Conflict of interest DR has received honoraria from Ariad, Bristol Myers Squibb, Novartis and Pfizer. J-MC has received honoraria from Ariad, Bristol Myers Squibb and Novartis. This article has not been supported by the pharmaceutical industry.

\section{References}

1. Druker BJ, Guilhot F, O'Brien SG, Gathmann I, Kantarjian H, Gattermann N, IRIS Investigators, et al. Five-year follow-up of patients receiving imatinib for chronic myeloid leukemia. $\mathrm{N}$ Engl J Med. 2006;355(23):2408-17.

2. Hochhaus A, Larson RA, Guilhot F, Radich JP, Branford S, Hughes TP, IRIS Investigators, et al. Long-term outcomes of imatinib treatment for chronic myeloid leukemia. N Engl J Med. 2017;376(10):917-27.

3. Hantschel O, Grebien F, Superti-Furga G. The growing arsenal of ATP-competitive and allosteric inhibitors of BCR-ABL. Cancer Res. 2012;72(19):4890-5.

4. Barrett AJ, Ito S. The role of stem cell transplantation for chronic myelogenous leukemia in the 21 st century. Blood. 2015;125(21):3230-5.
5. Rosti G, Castagnetti F, Gugliotta G, Baccarani M. Tyrosine kinase inhibitors in chronic myeloid leukaemia: which, when, for whom? Nat Rev Clin Oncol. 2017;14(3):141-54.

6. Gambacorti-Passerini C, Antolini L, Mahon FX, Guilhot F, Deininger M, Fava C, et al. Multicenter independent assessment of outcomes in chronic myeloid leukemia patients treated with imatinib. J Natl Cancer Inst. 2011;103(7):553-61.

7. Bower H, Björkholm M, Dickman PW, Höglund M, Lambert PC, Andersson TM. Life expectancy of patients with chronic myeloid leukemia approaches the life expectancy of the general population. J Clin Oncol. 2016;34(24):2851-7.

8. Graham SM, Jørgensen HG, Allan E, Pearson C, Alcorn MJ, Richmond L, et al. Primitive, quiescent, Philadelphia-positive stem cells from patients with chronic myeloid leukemia are insensitive to STI571 in vitro. Blood. 2002;99(1):319-25.

9. Corbin AS, Agarwal A, Loriaux M, Cortes J, Deininger MW, Druker BJ. Human chronic myeloid leukemia stem cells are insensitive to imatinib despite inhibition of BCR-ABL activity. J Clin Invest. 2011;121(1):396-409.

10. Chu S, McDonald T, Lin A, Chakraborty S, Huang Q, Snyder DS, et al. Persistence of leukemia stem cells in chronic myelogenous leukemia patients in prolonged remission with imatinib treatment. Blood. 2011;118(20):5565-72.

11. Hamilton A, Helgason GV, Schemionek M, Zhang B, Myssina $\mathrm{S}$, Allan EK, et al. Chronic myeloid leukemia stem cells are not dependent on Bcr-Abl kinase activity for their survival. Blood. 2012;119(6):1501-10.

12. Baccarani M, Deininger MW, Rosti G, Hochhaus A, Soverini $\mathrm{S}$, Apperley JF, et al. European LeukemiaNet recommendations for the management of chronic myeloid leukemia: 2013. Blood. 2013;122(6):872-84.

13. Rousselot P, Huguet F, Rea D, Legros L, Cayuela JM, Maarek O, et al. Imatinib mesylate discontinuation in patients with chronic myelogenous leukemia in complete molecular remission for more than 2 years. Blood. 2007;109(1):58-60.

14. Mahon FX, Réa D, Guilhot J, Guilhot F, Huguet F, Nicolini F, Intergroupe Français des Leucémies Myéloïdes Chroniques, et al. Discontinuation of imatinib in patients with chronic myeloid leukaemia who have maintained complete molecular remission for at least 2 years: the prospective, multicentre Stop Imatinib (STIM) trial. Lancet Oncol. 2010;11(11):1029-35.

15. Ross DM, Branford S, Seymour JF, Schwarer AP, Arthur C, Yeung DT, et al. Safety and efficacy of imatinib cessation for CML patients with stable undetectable minimal residual disease: results from the TWISTER study. Blood. 2013;122(4):515-22.

16. Etienne G, Guilhot J, Rea D, Rigal-Huguet F, Nicolini F, Charbonnier A, et al. Long-term follow-up of the French stop imatinib (STIM1) study in patients with chronic myeloid leukemia. J Clin Oncol. 2017;35(3):298-305.

17. Cross NC, White HE, Colomer D, Ehrencrona H, Foroni L, Gottardi E, et al. Laboratory recommendations for scoring deep molecular responses following treatment for chronic myeloid leukemia. Leukemia. 2015;29(5):999-1003.

18. Rousselot P, Charbonnier A, Cony-Makhoul P, Agape P, Nicolini $\mathrm{FE}$, Varet $\mathrm{B}$, et al. Loss of major molecular response as a trigger for restarting tyrosine kinase inhibitor therapy in patients with chronic-phase chronic myelogenous leukemia who have stopped imatinib after durable undetectable disease. J Clin Oncol. 2014;32(5):424-30.

19. Lee SE, Choi SY, Bang JH, Kim SH, Jang EJ, Byeun JY, et al. Predictive factors for successful imatinib cessation in chronic myeloid leukemia patients treated with imatinib. Am J Hematol. 2013;88(6):449-54.

20. Lee SE, Choi SY, Song HY, Kim SH, Choi MY, Park JS, et al. Imatinib withdrawal syndrome and longer duration of imatinib have a close association with a lower molecular relapse after 
treatment discontinuation: the KID study. Haematologica. 2016;101(6):717-23.

21. Mahon FX, Richter J, Guilhot J, Hjorth-Hansen H, Almeida A, Janssen JWM, et al. Cessation of tyrosine kinase inhibitors treatment in chronic myeloid leukemia patients with deep molecular response: results of the Euro-Ski trial. Blood. 2016;128(22): abstract 787.

22. Kantarjian HM, Giles F, Gattermann N, Bhalla K, Alimena G, Palandri F, et al. Nilotinib (formerly AMN107), a highly selective BCR-ABL tyrosine kinase inhibitor, is effective in patients with Philadelphia chromosome-positive chronic myelogenous leukemia in chronic phase following imatinib resistance and intolerance. Blood. 2007;110(10):3540-6.

23. Shah NP, Kantarjian HM, Kim DW, Réa D, DorlhiacLlacer PE, Milone JH, et al. Intermittent target inhibition with dasatinib $100 \mathrm{mg}$ once daily preserves efficacy and improves tolerability in imatinib-resistant and -intolerant chronic-phase chronic myeloid leukemia. J Clin Oncol. 2008;26(19):3204-12.

24. Hughes TP, Lipton JH, Spector N, Cervantes F, Pasquini R, Clementino NC, et al. Deep molecular responses achieved in patients with CML-CP who are switched to nilotinib after longterm imatinib. Blood. 2014;124(5):729-36.

25. Cortes JE, Saglio G, Kantarjian HM, Baccarani M, Mayer J, Boqué $\mathrm{C}$, et al. Final 5-year study results of DASISION: the dasatinib versus imatinib study in treatment-naïve chronic myeloid leukemia patients trial. J Clin Oncol. 2016;34(20):2333-40.

26. Hochhaus A, Saglio G, Hughes TP, Larson RA, Kim DW, Issaragrisil S, et al. Long-term benefits and risks of frontline nilotinib vs imatinib for chronic myeloid leukemia in chronic phase: 5-year update of the randomized ENESTnd trial. Leukemia. 2016;30(5):1044-54.

27. Imagawa J, Tanaka H, Okada M, Nakamae H, Hino M, Murai K, DADI Trial Group, et al. Discontinuation of dasatinib in patients with chronic myeloid leukaemia who have maintained deep molecular response for longer than 1 year (DADI trial): a multicentre phase 2 trial. Lancet Haematol. 2015;2(12):528-35.

28. Shah NP, Paquette R, Müller MC, Saussele S, Garcìa-Gutiérrez $\mathrm{V}$, Jiménez-Velasco A, et al. Treatment-free remission (TFR) in patients with chronic phase chronic myeloid leukemia (CML$\mathrm{CP}$ ) and in stable deep molecular response (DMR) to dasatinibthe Dasfree Study. Blood. 2016;128(22): abstract 1895.

29. Rea D, Nicolini FE, Tulliez M, Guilhot F, Guilhot J, GuerciBresler A, France Intergroupe des Leucémies Myéloïdes Chroniques, et al. Discontinuation of dasatinib or nilotinib in chronic myeloid leukemia: interim analysis of the STOP 2G-TKI study. Blood. 2017;129(7):846-54.

30. Hughes TP, Boquimpani CM, Takahashi N, Benyamini N, Clementino NCD, Shuvaev V, et al. Treatment-free remission in patients with chronic myeloid leukemia in chronic phase according to reasons for switching from imatinib to nilotinib: subgroup analysis from ENESTop. Blood. 2016;128(22): abstract 792.

31. Hochhaus A, Masszi T, Giles FJ, Radich JP, Ross DM, Gómez Casares MT, et al. Treatment-free remission (TFR) in patients (pts) with chronic myeloid leukemia in chronic phase (CML-CP) treated with frontline nilotinib: results from the ENESTFreedom study. J Clin Oncol. 2016; 34(suppl; abstr 7001).

32. Kumagai T, Nakaseko C, Nishiwaki K, Yoshida C, Ohashi K, Takezako N, et al. Discontinuation of dasatinib after deep molecular response for over 2 years in patients with chronic myelogenous leukemia and the unique profiles of lymphocyte subsets for successful discontinuation: a prospective, multicenter Japanese trial (D-STOP trial). Blood. 2016;128(22): abstract 791.

33. Kadowaki N, Kawaguchi T, Kurod J, Nakamae H, Matsumura I, Miyamoto $\mathrm{T}$, et al. Discontinuation of nilotinib in patients with chronic myeloid leukemia who have maintained deep molecular responses for at least 2 years: a multicenter phase 2 stop nilotinib (nilst) trial. Blood. 2016; 128(22): abstract 790.

34. Takahashi N, Nakaseko C, Nishiwaki K, Wakita H. Two-year consolidation by nilotinib is associated with successful treatment free remission in chronic myeloid leukemia with $\mathrm{MR}^{4.5}$ : subgroup analysis from STAT2 trial in Japan. Blood. 2016;128(22): abstract 1889.

35. Rea D, Rosti G, Cross NCP, Hellmann A, Niederwieser D, Pungolino E, et al. ENESTPath: A phase 3 study to assess the effect of nilotinib treatment duration on treatment-free remission (TFR) in patients with chronic myeloid leukemia in chronic phase (CML-CP) previously treated with imatinib: 24-month analysis of the first 300 patients in the induction/consolidation phase. Blood. 2016;128(22): abstract 3094.

36. Parker WT, Yeoman AL, Jamison BA, Yeung DT, Scott HS, Hughes TP, et al. BCR-ABL1 kinase domain mutations may persist at very low levels for many years and lead to subsequent TKI resistance. Br J Cancer. 2013;109(6):1593-8.

37. Jabbour E, Kantarjian H, O'Brien S, Rios MB, Abruzzo L, Verstovsek $\mathrm{S}$, et al. Sudden blastic transformation in patients with chronic myeloid leukemia treated with imatinib mesylate. Blood. 2006;107(2):480-2.

38. Latagliata R, Romano A, Mancini M, Breccia M, Carmosino I, Vozella F, et al. Discontinuation of alpha-interferon treatment in patients with chronic myeloid leukemia in long-lasting complete molecular response. Leuk Lymphoma. 2016;57(1):99-102.

39. Yong AS, Goldman JM. Relapse of chronic myeloid leukaemia 14 years after allogeneic bone marrow transplantation. Bone Marrow Transplant. 1999;23(8):827-8.

40. Park JS, Lee SE, Jeong SH, Jang EJ, Choi MY, Kim HJ, et al. Change of health-related profiles after imatinib cessation in chronic phase chronic myeloid leukemia patients. Leuk Lymphoma. 2016;57(2):341-7.

41. Richter J, Söderlund S, Lübking A, Dreimane A, Lotfi K, Markevärn $\mathrm{B}$, et al. Musculoskeletal pain in patients with chronic myeloid leukemia after discontinuation of imatinib: a tyrosine kinase inhibitor withdrawal syndrome? J Clin Oncol. 2014;32(25):2821-3.

42. Laneuville P. Stopping second-generation TKIs in CML. Blood. 2017;129(7):805-6.

43. Mori S, Vagge E, le Coutre P, Abruzzese E, Martino B, Pungolino E, et al. Age and $\mathrm{dPCR}$ can predict relapse in CML patients who discontinued imatinib: the ISAV study. Am J Hematol. 2015;90(10):910-4.

44. Zhang B, Li L, Ho Y, Li M, Marcucci G, Tong W, et al. Heterogeneity of leukemia-initiating capacity of chronic myelogenous leukemia stem cells. J Clin Invest. 2016;126(3):975-91.

45. Hughes TP, Ross DM. Moving treatment-free remission into mainstream clinical practice in CML. Blood. 2016;128(1):17-23.

46. Steegmann JL, Baccarani M, Breccia M, Casado LF, GarcíaGutiérrez V, Hochhaus A, et al. European LeukemiaNet recommendations for the management and avoidance of adverse events of treatment in chronic myeloid leukaemia. Leukemia. 2016;30(8):1648-71.

47. Abruzzese E, Trawinska MM, de Fabritiis P, Baccarani M. Management of pregnant chronic myeloid leukemia patients. Expert Rev Hematol. 2016;9(8):781-91.

48. Takahashi N, Kyo T, Maeda Y, Sugihara T, Usuki K, Kawaguchi T, et al. Discontinuation of imatinib in Japanese patients with chronic myeloid leukemia. Haematologica. 2012;97(6):903-6.

49. Benjamini O, Kantarjian H, Rios MB, Jabbour E, O'Brien S, Jain $\mathrm{P}$, et al. Patient-driven discontinuation of tyrosine kinase inhibitors: single institution experience. Leuk Lymphoma. 2014;55(12):2879-86.

50. Rege-Cambrin G, Fava C, Dogliotti I, Berchialla P, Gottardi E, Cerrano M, et al. Imatinib discontinuation in chronic myeloid 
leukemia: a retrospective analysis on patients in clinical practice. Haematologica 2015;100(Supplement 1): abstract: P603.

51. Kong JH, Winton EF, Heffner LT, Chen Z, Langston AA, Hill $\mathrm{B}$, et al. Does the frequency of molecular monitoring after tyrosine kinase inhibitor discontinuation affect outcomes of patients with chronic myeloid leukemia? Cancer. 2017;. doi:10.1002/ cncr.30608.

52. Ferrero D, Cerrano M, Crisà E, Aguzzi C, Giai V, Boccadoro M. How many patients can proceed from chronic myeloid leukaemia diagnosis to deep molecular response and long-lasting imatinib discontinuation? A real life experience. Br J Haematol. 2017;176(4):669-71.

53. Sato T, Onai N, Yoshihara H, Arai F, Suda T, Ohteki T. Interferon regulatory factor- 2 protects quiescent hematopoietic stem cells from type I interferon-dependent exhaustion. Nat Med. 2009;15(6):696-700.

54. Essers MA, Offner S, Blanco-Bose WE, Waibler Z, Kalinke U, Duchosal MA, et al. IFNalpha activates dormant haematopoietic stem cells in vivo. Nature. 2009;458(7240):904-8.

55. Preudhomme C, Guilhot J, Nicolini FE, Guerci-Bresler A, RigalHuguet F, Maloisel F, SPIRIT Investigators, France Intergroupe des Leucémies Myéloïdes Chroniques (Fi-LMC), et al. Imatinib plus peginterferon alfa-2a in chronic myeloid leukemia. N Engl J Med. 2010;363(26):2511-21.

56. Legros L, Rousselot P, Giraudier S, Tulliez M, Huguet F, Nicolini FE, et al. Second attempt to discontinue imatinib in CP-CML patients with a second sustained complete molecular response. Blood. 2012;120(9):1959-60.

57. Holyoake TL, Vetrie D. The chronic myeloid leukemia stem cell: stemming the tide of persistence. Blood. 2017;129(12):1595-606.

58. Rea D, Dulphy N, Henry G, Guilhot J, Guilhot F, Nicolini FE, et al. Low natural killer (NK) cell counts and functionality are associated with molecular relapse after imatinib discontinuation in patients (pts) with chronic phase (CP)-chronic myeloid leukemia (CML) with undetectable BCR-ABL transcripts for at least 2 years: preliminary results from Immunostim, on behalf of STIM Investigators (abstract). Blood. 2013;122(21): abstract 856.

59. Ilander M, Olsson-Strömberg U, Schlums H, Guilhot J, Brück O, Lähteenmäki $\mathrm{H}$, et al. Increased proportion of mature NK cells is associated with successful imatinib discontinuation in chronic myeloid leukemia. Leukemia. 2016;. doi:10.1038/leu.2016.360. 